\title{
2012/33
}

\section{Pareto optimality of the golden rule equilibrium in an overlapping generations model with production and transfers}

Jean-François Mertens and Anna Rubinchik

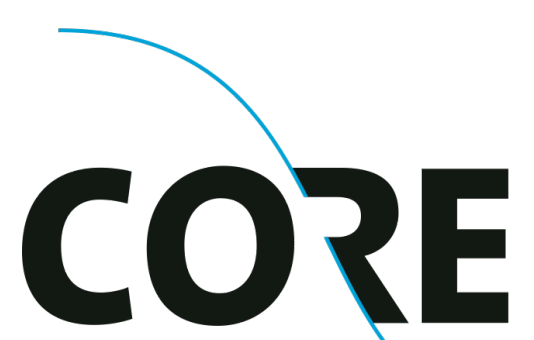

\section{DISCUSSION PAPER}

Center for Operations Research and Econometrics

Voie du Roman Pays, 34 B-1348 Louvain-la-Neuve Belgium http://www.uclouvain.be/core 


\title{
CORE DISCUSSION PAPER \\ $2012 / 33$
}

\section{Pareto optimality of the golden rule equilibrium in an overlapping generations model with production and transfers}

\section{Jean-François MERTENS ${ }^{1}$ and Anna RUBINCHIK ${ }^{2}$}

\begin{abstract}
August 2012
Abstract

The main result is that the golden rule equilibrium (GRE) is Pareto optimal (in the classical sense) in an overlapping generations (OG) model with constant-returns-to-scale production, transfers, arbitrary life-time productivity and CES instantaneous felicity. In addition, we extend Cass and Yaari's [10] equivalence between efficiency (aggregate consumption dominance) and the present value dominance (with evaluation made using a candidate equilibrium price path).

Keywords: infinite economies, overlapping generations, exogenous growth, golden rule equilibrium.
\end{abstract}

JEL classification: D50, E20

MSC classification: 91B62, 91B50

\footnotetext{
${ }^{1}$ Université catholique de Louvain, CORE, B-1348 Louvain-la-Neuve, Belgium.

${ }^{2}$ Department of Economics, University of Haifa, 31905 Haifa, Israël. E-mail: annarubinchik@gmail.com.

We would like to thank for its hospitality the Center for Rationality in Jerusalem and CORE. The scientific responsibility is assumed by the authors.
} 


\section{InTRODUCTION}

We prove Pareto optimality of a golden rule equilibrium (GRE) in an overlapping generations (OG) model in continuous time with production (and transfers). From the very start ([1], [15], [19]) the OG models were known to have non-Pareto competitive equilbria, and since then the question of existence of a Pareto optimal equilibrium allocation in such models has been open. ${ }^{1}$ The literature provides both positive answers, e.g., [3], [8], [18], [12], [5]; as well as negative ones, e.g., [9]. It is crucial, however, that the traditional optimality criterion is weak ("one-sided"), requiring an equilibrium allocation not be dominated only starting from some point in time (with the exception of, e.g., [14] studying endowment economies). ${ }^{2}$ Although "irreversibility of time" might sound appealing (and so it is tempting to "forget about the past") using the weak notion of optimality one must accept as optimal, a.o., all stationary equilbria with "under-accumulation" of capital, which are everywhere dominated by the GRE. Hence, we turn back to the classical Pareto criterion and show, building upon a careful characterisation of equilibria of the model in [16] (e.g., not requiring neither prices nor capital to be everywhere positive), that GRE is Pareto. This is our first theorem.

The closest model is, probably, by Cass and Yaari [10], who analyse Pareto optimality and Malinvaud's [15] efficiency (dominance by an aggregate consumption path) in an economy with production in continuous time with logarithmic instantaneous felicities, constant life-time productivity, and no transfers. However, the equilibrium characterisation employed there is constricted by a specific price path, ${ }^{3}$ and the notion of Pareto (and efficient) allocation, too, is one-sided (in addition, for Pareto, limiting alternative paths to only those that improve the stream of "instantaneous felicities" at any point in the life-time of an individual).

The second theorem extends Cass and Yaari's [10, thm. 1]: a feasible consumption is the highest if and only if its present value is. It is provided, in particular, to highlight the intricacies of proving efficiency for an unrestricted set of feasible paths in a model with transfers.

Our last result, lemma 1, demonstrates that the usual criteria for efficiency imply that the net assets are zero, which, a.o. is true for the balanced growth equilibria (BGE) that are not GRE, hence typically are neither efficient nor Pareto.

\section{THE MODEL}

Consider the basic OG model from [16]: the life span of any individual born at $x \in \mathbb{R}$ is $[0,1]$;

$$
U_{x}=\int_{0}^{1} e^{-\beta s} u\left(\hat{c}_{x, s}\right) d s, \text { with } u(z)=\frac{z^{1-\frac{1}{\sigma}}}{1-\frac{1}{\sigma}}, \text { for } \sigma \neq 1
$$

is his life-time utility defined over the set of individual consumption plans $\hat{c}_{x, s}$, $\overline{\mathbb{R}}_{+}$-valued Lebesgue-measurable functions of age $s$ for every $x$. Individual life-time income consists of individual- and age-specific transfers.

Aggregate total productive labour available at $t$ is:

$$
L_{t}=N_{0} e^{\gamma t} \int_{t-1}^{t} \zeta_{t-x} e^{\nu x} d x=N_{0} e^{(\gamma+\nu) t} \int_{0}^{1} \zeta_{s} e^{-\nu s} d s
$$

\footnotetext{
${ }^{1}$ The "optimum property of the biological interest rate" (in an exchange discrete model with two period life-cycle) has been established by Samuelson [19], but optimality of an allocation there requires it not to be dominated by just another stationary one (with a constant interest rate), and hence is far weaker than the classical Pareto criterion.

${ }^{2}$ The characterisation of optimal no-trade equilibria in [14] inspired the initial direction we took in proving our first theorem.

${ }^{3}$ The question of existence is omitted.
} 
where $N_{x} d x \stackrel{\text { def }}{=} N_{0} e^{\nu x} d x\left(N_{0}>0\right)$ individuals get born in $[x, x+d x], \forall x \in \mathbb{R}, \zeta_{s}$ is individual productivity, non-negative over a life-span $[0,1]$, and zero elsewhere, $\nu$ is the rate of the population growth and $\gamma$ is the per-capita productivity growth. Aggregate capital evolves according to the differential equation $K_{t}^{\prime}=I_{t}-\delta K_{t}$ with aggregate investment $I_{t}$ and depreciation factor $\delta$.

There is a constant-returns-to-scale instantaneous production function $F\left(K_{t}, L_{t}\right)$, $F: \mathbb{R}_{+}^{2} \rightarrow \mathbb{R}_{+}$concave, continuous, positively homogeneous of degree 1 , and differentiable on $\mathbb{R}_{++}^{2} \cdot f(x) \stackrel{\text { def }}{=} F(x, 1)$. The production set is the closed convex cone spanned by the production function, free-disposal, and 2-way transformations of output into consumption and investment.

Assumption 1 (Initial Condition). For any feasible path $K, e^{\left(\delta-f_{\infty}^{\prime}\right) t} K_{t}$ converges exponentially to 0 at $-\infty$, where $f_{\infty}^{\prime} \stackrel{\text { def }}{=} \lim _{x \rightarrow \infty} \frac{f(x)}{x}$.

The word "exponentially" can be dropped here iff $\int_{1}^{\infty} \frac{f(x)-x f_{\infty}^{\prime}}{x^{2}} d x<\infty$.

Notation 2.1. $\quad$ (i) $E_{t, s}=\frac{N_{t-s} \omega_{t-s, s}}{L_{t}},{ }^{4} \Omega_{t}=\int_{0}^{1} E_{t, s} d s, c_{t, s}^{\odot}=\frac{N_{t-s} \hat{c}_{t-s, s}}{L_{t}}$, (so $\left.c_{t}=\int_{0}^{1} c_{t, s}^{\odot} d s\right), k_{t}=\frac{K_{t}}{L_{t}}, y_{t}=\frac{Y_{t}}{L_{t}}, i_{t}=\frac{I_{t}}{L_{t}}, c_{t}=\frac{C_{t}}{L_{t}}$.

(ii) $\varphi_{s}=\frac{e^{-\nu s} \zeta_{s}}{\int_{0}^{1} e^{-\nu u} \zeta_{u} d u}, \eta=(\gamma+\nu)(1-\sigma)+\beta \sigma, R=\gamma+\nu+\delta, \Phi(x)=\frac{e^{x}-1}{x}$;

(iii) $\phi(k) \stackrel{0}{=} f(k)-R k, \mathfrak{r}_{t}=R-f^{\prime}\left(k_{t}\right)\left(=-\phi^{\prime}\left(k_{t}\right)\right)$;

(iv) For $h: \mathbb{R} \rightarrow \mathbb{R},\|h\|_{\infty, 1}=\sup _{x} \int_{x-1}^{x}|h(t)| d t$, and $\|E\|_{\infty, 1}=\sup _{x} \int_{x-1}^{x} \int\left|E_{t, s}\right| d s d t$.

Assumption 2. $\|E\|_{\infty, 1}<\infty .^{5} f_{\infty}^{\prime}<R$, and $\exists x: f(x)>R x$; i.e., $F(1,0)<R<$ $F(1, \infty)$.

Definition 1. (i) Stationary endowments mean $\omega_{x, s}=e^{\gamma x} \omega_{s}$.

(ii) A balanced growth equilibrium (BGE) is an equilibrium of an economy with stationary endowments, such that $K_{t}$ is an exponential function of time.

(iii) A BGE is a golden rule equilibrium (GRE) if $\forall t, f\left(k_{t}\right)-R k_{t}=\max _{k}(f(k)-R k)$.

Notation 2.2. For stationary endowments, we use $E_{s}=\frac{e^{-(\gamma+\nu) s} \omega_{s}}{\int_{0}^{1} \zeta_{s} e^{-\nu s} d s}$ and $\Omega=\int E_{s} d s$.

Definition 2. (i) A plan or allocation is by definition feasible: individual consumptions belong to the consumption sets, production plans lie in the production sets, and there is material balance,

$$
c_{t}+i_{t} \leq y_{t}+\Omega_{t}
$$

Equivalently, all equilibrium conditions are satisfied except for optimisation by firms and individuals.

A utility profile $U_{x}$, or a capital-consumption path $\left(K_{t}, C_{t}\right)$, etc., is feasible if it is induced by some feasible plan.

(ii) An allocation is Pareto optimal, resp., efficient, if it induces a maximal point (with the usual order on $\overline{\mathbb{R}}$-valued Lebesgue-measurable functions) in the set of feasible utility profiles $U_{x}$, resp., aggregate consumption $C_{t}$.

Comment 1. The concept of efficiency (due to [15]), as distinct from Pareto-optimality, has 2 potential interests: the first is to enable to distinguish sources of non-optimality as being in the finite lives of the firms (production sector) or of the consumers; the other is that, while Pareto-optimality is a tempting definition of GRE for economies with a single type of consumer, independently of the number of goods of all sorts [17], it is efficiency that would seem a natural candidate in the case of a single consumption good, but many types of agents and of all other goods.

\footnotetext{
${ }^{4} E_{t, s}$ (resp., $c_{t, s}^{\odot}$ ) is the normalised [per unit of productive labour at time $t$ ] aggregate endowment (resp. consumption) at time $t$ of individuals of age $s$.

${ }^{5}$ So, individual endowments are not necessarily bounded, but have to be locally integrable.
} 
Remark 2. The material balance equation (1) and the capital accumulation equation derived in [16, cor.2] imply that for any feasible path $k_{t}^{\prime} \leq \phi\left(k_{t}\right)+\Omega_{t}-c_{t}$.

Next, we reproduce [16, cor. 15]:

Corollary 1. Assume $f^{\prime}(0)>R$ and the endowments are stationary.

(i) If $\Omega+\sup _{k \geq 0}(f(k)-R k) \geq 0$ then there exists a GRE.

(ii) Denote GRE variables with superscript $G$. The GRE are the solutions of
(a) $f^{\prime}\left(k^{G}\right)=R$, so $\mathfrak{r}^{G}=0$
(b) $y^{G}=f\left(k^{G}\right)$
(c) $i^{G}=R k^{G}$
(d) $c^{G}=\Omega+f\left(k^{G}\right)-k^{G} f^{\prime}\left(k^{G}\right)$
(e) $p_{t}^{G}=p_{0}^{G} e^{-(\gamma+\nu) t}, p^{I}=p^{C}=p^{Y}=p^{G}$
(f) $w_{t}^{G}=p_{0}^{G} e^{-\nu t}\left(y^{G}-R k^{G}\right), r_{t}^{G}=R p_{t}^{G}$

(iii) Inequality (i) is necessary for the existence of a feasible path.

Proof. All the claims of the corollary but the last one, iii, are proved in [16]. The last claim clearly follows from thm. 1 below, but can be shown to hold without its additional assumption.

(iii): First, by assumption $2, \sup _{k \geq 0}(f(k)-R k)>0$ is attained at a finite $k$, and by point (iid) of the corollary, $c^{G}=\sup _{k \geq 0} \phi(k)+\Omega$. If inequality (i) is violated, there is $\epsilon>0$ such that $c^{G}<-\epsilon<0$.

Assume to the contrary that such economy can have a feasible path, so, $c_{t} \geq 0$ for all $t$. It follows that $c^{G}-c_{t}<-\epsilon$ for all $t$. This implies, by remark 2 , that $k_{t}^{\prime} \leq$ $\phi\left(k_{t}\right)+\Omega-c_{t}<\phi\left(k_{t}\right)+\Omega-c^{G}-\epsilon \leq-\epsilon$, for all $t$, therefore contradicting $k_{t} \geq 0$.

\section{The MAIN Result}

Theorem 1. Assume $\lim \inf _{k \rightarrow k^{G}} \frac{\phi\left(k^{G}\right)-\phi(k)}{\left(k-k^{G}\right)^{2}}>0$. Then a GRE allocation is Pareto optimal.

Proof. By [16, lemma 1], it is sufficient to prove the result in the reduced economy, $\mathfrak{E}^{\prime}$ where $\gamma=\nu=0, N_{0}=1, \int_{0}^{1} \zeta d s=1$.

We show a bit more: that any plan $\left(\hat{c}_{x, s}, c_{t}, k_{t}, y_{t}, i_{t}\right)$ with $U_{x} \stackrel{\text { def }}{=} U\left(\hat{c}_{x}\right) \geq U^{G}$ a.e. equals the GRE a.e., where $U^{G}=u\left(c^{G}\right)[\Phi(-\eta)]^{\frac{1}{\sigma}}$ is the life-time utility of any individual in GRE.

Step 1. $C_{x} \stackrel{\text { def }}{=} \int_{0}^{1} \hat{c}_{x, s} d s \geq c^{G}$ for a.e. $x \in \mathbb{R}$.

Proof. Neglect the set of $x$ where $U_{x}<U^{G}$ (which is negligible). Then, since $\gamma=\nu=0$, the price $p_{t}$ is constant in GRE and can be set to 1 , the integral is the cost of a bundle in GRE prices. And in GRE the life-time income is the sum of transfers, $\int_{0}^{1} E_{s} d s=\Omega$, and the wage income, $\int_{0}^{1} w_{x+s}^{G} \zeta_{s} d s$, which is $=y^{G}-R k^{G}$, by cor. 1.iif, and since $f^{\prime}\left(k^{G}\right)=R$ by the same corollary (cond. iia), the income is equal to $c^{G}$ (by cond. iid). Since $\hat{c}_{x}+\varepsilon \geq \varepsilon, U\left(\hat{c}_{x}+\varepsilon\right)>-\infty$, so since $U\left(\hat{c}_{x}\right) \geq U\left(\hat{c}^{G}\right)$, $\hat{c}_{x}+\varepsilon$ is strictly preferred to $\hat{c}^{G}$, thus $\int_{0}^{1}\left(\hat{c}_{x, s}+\varepsilon\right) d s>c^{G}$, hence the result.

Step 2. $A_{t} \stackrel{\text { def }}{=} \iint_{0 \leq t-{ }_{b} x<s \leq 1} \hat{c}_{x, s} d x d s$ is a primitive of $C_{t}-c_{t} \in L_{1}^{\text {loc }}$, and is bounded: $\exists \kappa \in \mathbb{R}, \forall a, b \in \mathbb{R}: \int_{a}^{b}\left(C_{t}-c_{t}\right) d t=A_{b}-A_{a} \leq \kappa$.

Proof. Suffices to do the proof for $a \leq b$. Let $X_{t}=\{(x, s) \mid 0 \leq t-x<s \leq 1\}$, so $A_{t}=\iint_{X_{t}} \hat{c}$. By [16, prop.1.c], $\hat{c}$ is integrable on any bounded subset of $\mathbb{R} \times[0,1]$. By Fubini's theorem $\int_{a}^{b} C_{x} d x$ is the integral of $\hat{c}$ on a bounded set $D$ $\left(=\{a<x \leq b, 0 \leq s \leq 1\}\right.$, interpreting $\int_{a}^{b}$ as $\left.\int_{[a, b]}\right)$, and $\int_{a}^{b} c_{t} d t$ is that on another bounded set $D^{\prime}(=\{a<x+s \leq b, 0 \leq s \leq 1\})$, and so the difference of the integrals is well-defined, and equals the integral on $D \backslash D^{\prime}=X_{b} \backslash X_{a}$ minus the integral on $D^{\prime} \backslash D=X_{a} \backslash X_{b}$, i.e., $A_{b}-A_{a}$, which is again bounded by [16, prop. 1.c]. 
Step 3. $\exists \kappa \in \mathbb{R}: \forall a \leq b, \int_{a}^{b}\left(c_{t}-\Omega-\phi\left(k_{t}\right)\right) d t \leq k_{a}-k_{b} \leq \kappa$.

Proof. By feasibility, $c_{t} \leq f\left(k_{t}\right)-i_{t}+\Omega$, and $k_{b}-k_{a}=\int_{a}^{b}\left(i_{t}-R k_{t}\right) d t$. Combining the two along with the bounds on $k_{t}$ from [16, prop. 1.a] we get the result.

\section{Step 4.}

(1) $\int_{-\infty}^{\infty}\left(C_{t}-\Omega-\phi\left(k_{t}\right)\right) d t \leq \liminf _{a, b \rightarrow \infty}\left[\left(A_{b}-A_{-a}\right)-\left(k_{b}-k_{-a}\right)\right] \quad$ where $C_{t}-\Omega \geq \phi\left(k_{t}\right)$

(3) $\int_{-\infty}^{\infty}\left(\phi\left(k^{G}\right)-\phi\left(k_{t}\right)\right) d t \leq \kappa$

where $C_{t} \geq c^{G}$

where $\phi\left(k^{G}\right) \geq \phi\left(k_{t}\right)$

Proof. Summing the inequalities of steps 2 and 3 implies that $\exists \kappa, \forall a \leq b$ :

$$
\int_{a}^{b}\left(C_{t}-\Omega-\phi\left(k_{t}\right)\right) d t \leq A_{b}-A_{a}+k_{a}-k_{b} \leq \kappa
$$

By step $1, C_{t} \geq c^{G}$ a.e. Also $c^{G}-\Omega=\phi\left(k^{G}\right) \geq \phi\left(k_{t}\right)$, since $\phi\left(k^{G}\right)=\max _{k} \phi(k)$. Thus we get the inequalities, by monotone convergence.

Our purpose in the following is to show that, in (1), both $A_{b}-A_{a}$ and $k_{b}-k_{a}$ converge to 0 , so that the integrals in steps 2 and 3 are 0 , when viewed as improper Lebesgue integrals from $-\infty$ to $+\infty$ (and forgetting the inequality due to possible free-disposal in the latter). Since the integral in (1) is the sum of those in (3) and (2), both of which have non-negative integrands, the conclusion will follow.

Step 5. ||$k-k^{G} \|_{2}<\infty$; so, $\lim \inf \left|k_{t}-k^{G}\right|=0$ when $t \rightarrow \infty$ and when $t \rightarrow-\infty$.

Proof. By [16, prop. 1.a] any feasible capital path is bounded by some $\kappa$. Majorise $\phi(k)$ on $[0, \kappa]$ by $\phi\left(k^{G}\right)-\varepsilon\left(k-k^{G}\right)^{2}$ (using unimodality of $\phi$, compactness of the interval, and the assumption $\left.\lim \inf _{k \rightarrow k^{G}} \frac{\phi\left(k^{G}\right)-\phi(k)}{\left(k-k^{G}\right)^{2}}>0\right)$. Then, by (3),

$$
\left\|k_{t}-k^{G}\right\|_{2}^{2}=\int_{-\infty}^{\infty}\left(k_{t}-k^{G}\right)^{2} d t \leq \kappa / \varepsilon
$$

Step 6. Let $F(x)=\sqrt{x}$ for $0 \leq x \leq 1,=\frac{1}{2} x+\frac{1}{2}$ for $x \geq 1$. $\exists z:\left\|\hat{c}_{x}-\hat{c}^{G}\right\|_{1} \leq$ $z F\left(C_{x}-c^{G}\right)$ a.e. in $x$.

Proof. Assume first $c^{G}>0$. Let $G(x)=F^{-1}(|x|)$. Then $\exists \varepsilon>0$ s.t., $\forall s \in[0,1]$ and $\forall x>0, e^{-\frac{\eta}{\sigma} s}\left(u(x)-u\left(\hat{c}_{s}^{G}\right)\right) \leq\left(\frac{\Phi(-\eta)}{c^{G}}\right)^{\frac{1}{\sigma}}\left(x-\hat{c}_{s}^{G}\right)-\varepsilon G\left(\frac{x-\hat{c}_{s}^{G}}{c^{G}}\right)$, because $\hat{c}_{s}^{G}$ varies in a compact interval, not containing 0 (since $c^{G}>0$ ), the first derivative of $e^{-\frac{\eta}{\sigma} s} u(\cdot)$ at $\hat{c}_{s}^{G}$ equals $\left(\frac{\Phi(-\eta)}{c^{G}}\right)^{\frac{1}{\sigma}}$, and the second is continuous and $<0 .^{6}$

Use this to bound $e^{-\frac{\eta}{\sigma} s} u\left(\hat{c}_{x, s}\right): U_{x}-U^{G} \geq 0$ yields $\int_{0}^{1} G\left(\frac{\hat{c}_{x, s}-\hat{c}_{s}^{G}}{c^{G}}\right) d s \leq \kappa\left(C_{x}-c^{G}\right)$.

Then, since $G(x)=G(|x|)$, Jensen's inequality (convexity of $G$ ) yields (using $\mathrm{E}$ for expectation) that, for a random variable $X$, in this case $\frac{\hat{c}_{x, s}-\hat{c}_{s}^{G}}{c^{G}}$ where $s$ has the uniform distribution on $[0,1], \mathrm{E} G(X)=\mathrm{E} G(|X|) \geq G(\mathrm{E}|X|)$. Thus, since $G$ is monotone on $\mathbb{R}_{+}, \mathrm{E}|X| \leq G^{-1}(\mathrm{E} G(X))$. Hence $\left\|\hat{c}_{x}(\cdot)-\hat{c}^{G}(\cdot)\right\|_{1} \leq$ $c^{G} F\left(\int_{0}^{1} G\left(\frac{\hat{c}_{x, s}-\hat{c}_{s}^{G}}{c^{G}}\right) d s\right) \leq c^{G} F\left(\kappa\left(C_{x}-c^{G}\right)\right)$. Choose then $z$ s.t. $\forall x, z F(x) \geq c^{G} F(\kappa x)$, which exists by concavity of $F$.

If $c^{G}=0$, i.e., $\Omega=-\phi\left(k^{G}\right)$, then the integrand in step 3 is the sum of the 2 non-negative functions $c_{t}$ and $\phi\left(k^{G}\right)-\phi\left(k_{t}\right)$; since by step $5 \lim \inf \left(k_{a}-k_{b}\right) \leq 0$ when $a \rightarrow-\infty, b \rightarrow \infty$, both functions are 0 a.e. by monotone convergence. And

\footnotetext{
${ }^{6}$ Indeed, by continuity $u^{\prime \prime}<-2 \varepsilon_{0}$ on that compact interval, thus also $e^{-\frac{\eta}{\sigma} s} u^{\prime \prime}$. Hence, $\forall \varepsilon<\varepsilon_{0}$, the claimed upper bound majorises the left-hand member on that compact interval $I$, even with $G(x)=x^{2}$. By compactness (and $u^{\prime \prime}<0$ ), decreasing $\varepsilon_{0}$ further will also yield the inequality everywhere between 0 and that compact interval (in fact, for our specific $u$, this is not needed, because $u^{\prime \prime}$ decreases), and on an interval of length $c^{G}$ to the right of $I$, ending say at $x_{0}$. $u^{\prime \prime}<0$ implies that $\exists \delta>0 \forall s \forall x \geq x_{0}: e^{-\frac{\eta}{\sigma} s} u^{\prime}(x)<e^{-\frac{\eta}{\sigma} s} u^{\prime}\left(c_{s}^{G}\right)-2 \delta$. Since the $G$ term is linear with slope 2 in that region, any $\varepsilon \leq \min \left\{\varepsilon_{0}, \delta\right\}$ will ensure that the slope of the upper bound is greater that that of the left hand member $\forall x \geq x_{0}$. Therefore, the inequality extends also everywhere to the right of $x_{0}$.
} 
by Fubini $c_{t}=0$ a.e. implies $\hat{c}_{x, s}=0$ a.e., hence $C_{x}=0$ a.e. Thus, a.e. in $x$, $\hat{c}_{x, s}=0=\hat{c}_{s}^{G} d s$-a.e.: $\left\|\hat{c}_{x}-\hat{c}^{G}\right\|_{1}=0$.

Step 7. $A_{t} \underset{|t| \rightarrow \infty}{\longrightarrow} A^{G}$ : the primitive $A_{t}$ is $\geq 0$ and $A_{t}-A^{G} \in C_{0}$, with $A^{G}=$ $\frac{1}{\eta}\left(1-\frac{1}{\Phi(\eta)}\right)$.

Proof. $\left|A_{t}-A^{G}\right| \leq \iint_{0 \leq t-x<s \leq 1}\left|\hat{c}_{x, s}-\hat{c}_{s}^{G}\right| d x d s \leq \int_{t-1}^{t} \int_{0}^{1}\left|\hat{c}_{x, s}-\hat{c}_{s}^{G}\right| d s d x=\int_{t-1}^{t} \| \hat{c}_{x}(\cdot)-$ $\hat{c}^{G}(\cdot) \|_{1} d x \leq z \int_{t-1}^{t} F\left(C_{x}-c^{G}\right) d x$, by step $6, \leq z F\left(\int_{t-1}^{t}\left(C_{x}-c^{G}\right) d x\right)$ by Jensen's inequality. By eq. (2), $\forall \varepsilon>0, \exists T: \int_{\complement[-T, T]}\left(C_{x}-c^{G}\right) d x \leq \varepsilon$. Thus for $|t| \geq T+1$, $\left|A_{t}-A^{G}\right| \leq z F(\varepsilon)$.

Step 8. The allocation equals the GRE allocation.

Proof. By step $5, \lim _{\inf } \rightarrow \pm \infty\left|k_{t}-k^{G}\right|=0$, so by step 7 , the right-hand member in (1) is 0 . Since its integrand is the sum of the non-negative integrands in (2) and (3), it follows that $C_{t}=c^{G}$ and $k_{t}=k^{G}$ a.e., so $k_{t}=k^{G} \forall t$, by continuity. By step 6 , $C_{t}=c^{G}$ a.e. implies $\hat{c}_{x, s}=\hat{c}_{s}^{G}$ a.e., and thus $U_{x}=U^{G}$ a.e. too, and $c_{t}=c^{G}$ a.e. by Fubini. And, by the capital accumulation equation (formally, use [16, cor. 2]), $k_{t}=k^{G}$ implies $i_{t}=i^{G}=R k^{G}$ a.e., so, by material balance $y_{t}=y^{G}$ a.e.: there is no unemployment and no free-disposal, and the allocation equals the GRE allocation a.e.

\section{CASS AND YAARI'S NECESSARY AND SUFFICIENT CONDITION FOR EFFICIENCY}

The main idea of the proof stems from Cass and Yaari [10, thm. 1 p. 264].

Proposition 1. If $f$ is strictly concave and $C$ is efficient, there is a unique $K_{t}$ s.t. $\left(K_{t}, C_{t}\right)$ is feasible.

Proof. Follows from the strict concavity of $f(k)$.

Theorem 2. Assume $f$ is strictly concave. Let $C$ be feasible.

If for every feasible $\tilde{C}$ there exists a feasible $(K, C)$ s.t., for any $t_{0}$ large enough and $p_{t}=\exp \left[\int_{t_{0}}^{t}\left(\delta-f^{\prime}\left(k_{s}\right)\right) d s\right], \liminf _{b,-a \rightarrow \infty} \int_{* a}^{b} p_{t}\left(\tilde{C}_{t}-C_{t}\right) d t \leq 0$ (with $\int_{*}$ being the lower Lebesgue integral, and the usual convention $\infty \times 0=0$ for products, cf. [16, fn. 4]), then $C$ is efficient.

Conversely, if $C$ is efficient then for every feasible $\tilde{C}$ there exists a feasible $(K, C)$ s.t., for every $t_{0}$ and $p_{t}=\exp \left[\int_{t_{0}}^{t}\left(\delta-f^{\prime}\left(k_{s}\right)\right) d s\right], \lim \inf _{b,-a \rightarrow \infty} \int_{* a}^{b} p_{t}\left(\tilde{C}_{t}-C_{t}\right) d t \leq 0$.

Remark 3. By [16, prop. 1.c], $\tilde{C}-C$ is a.e. well-defined and finite, so the integrand is too.

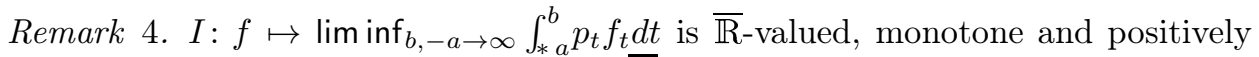
homogeneous of degree 1 on the set of all $\overline{\mathbb{R}}$-valued Lebesgue-measurable functions, with $I(f+g) \geq I(f)+I(g)$ ('concavity') when using $\infty-\infty=-\infty$ on both sides.

Remark 5. There is no 'justification' for the formula for $p_{t}$; even equilibrium relations (e.g., [16, thm.1.3], referring to [16, lemma 10.a5]) give it only modulo an additional function $\pi_{t}$, which can be neglected only when $K_{t}$ never vanishes while those possible zeros of $K_{t}$ are the main source of problems here.

An interpretation might be that efficient paths should behave as if $K_{t}$ never vanishes, i.e., recover as fast as possible after any zero (cf. the issue of multiplicity of solutions after a zero in [16, fn. 27]). A confirmation of this might be if one could prove from the theorem that, for efficient paths, $K_{t}$ vanishes only on a null set. 
Proof. By [16, lemma 1], it is sufficient to prove the result in the reduced economy, $\mathfrak{E}^{\prime}$ where $N_{0}=1, \int_{0}^{1} \zeta d s=1, \gamma=\nu=0$, so $\delta=R$.

If $c$ is not efficient, and $\tilde{c}$ a feasible improvement, choosing $t_{0}$ large enough to satisfy $\lambda\left\{t \leq t_{0} \mid \tilde{c}_{t}>c_{t}\right\}>0$ will ensure that $p_{t}>0$ on this set and thus that the inequality is violated.

Conversely, assume the inequality does not hold for some such price system $p_{t}$ and some $\tilde{c}$; then we have to show $c$ is not efficient. Write $c$ as $c^{0}$, and $\tilde{c}$ as $c^{1}$, corresponding resp. to $k^{0}$ (which defines $p$ ) and $k^{1}$.

By the hypothesis,

$$
\liminf _{b,-a \rightarrow \infty} I_{a, b}>0 \quad I_{a, b} \stackrel{\text { def }}{=} \int_{a}^{b} p_{t}\left(c_{t}^{1}-c_{t}^{0}\right) d t
$$

Since $\delta=R$ in $\mathfrak{E}^{\prime}$,

$$
p_{t}=\exp \left[\int_{t_{0}}^{t}\left(R-f^{\prime}\left(k_{s}^{0}\right)\right) d s\right]=\exp \left[-\int_{t_{0}}^{t} \phi^{\prime}\left(k_{s}^{0}\right) d s\right]
$$

Given the hypothesis, we construct the dominating capital-consumption path $\left(k^{2}, c^{2}\right)$ in a series of steps.

Step 1. Let $m \stackrel{\text { def }}{=} \inf \left\{t \mid p_{t}<\infty\right\}, M \stackrel{\text { def }}{=} \sup \left\{t \mid p_{t}>0\right\}$. Then $0<p_{t}<\infty$ on ]$m, M\left[, t \mapsto \phi^{\prime}\left(k_{t}^{0}\right) \in L_{1}^{\text {loc }}(] m, M[) ; M<\infty \Rightarrow k_{M}^{i}=0\right.$ and $m>-\infty \Rightarrow k_{m}^{i}=0$, both for $i \in\{0,1\}$. Finally, $\liminf _{a \searrow m, b} \operatorname{\nearrow i}_{M} I_{a, b}>0$.

Proof. Since $f^{\prime} \geq 0, R-f^{\prime}\left(k_{s}^{0}\right) \leq R$, so by (2) if $s<t, p_{t} \leq p_{s} e^{R(t-s)}$ thus, for $a<b$, the upward variation between $a$ and $b, \sum_{i: a<s_{i}<s_{i+1}<b}\left[p_{s_{i+1}}-p_{s_{i}}\right]^{+}$, is below $p_{a} e^{R(b-a)}$. Therefore, if $p_{t_{1}}<\infty$, then for any $t>t_{1}, p$ remains finite. Similarly, if $p_{t_{1}}=0$, it remains zero for all $t>t_{1}$. It follows that $\left\{t \mid 0<p_{t}<\infty\right\}$ is an interval $T_{1}$, with $t_{0} \in T_{1}$ (since $p_{t_{0}}=1$ by $(2)$ ), and that $p_{t}=\infty$ to the left of $T_{1}$ and $=0$ to the right. Hence $p_{t}$ has bounded variation on every interval $[a, b]$ s.t. $p_{a}<\infty$. Further, the restriction of $p_{t}$ to the closure of $T_{1}$ is continuous, by the monotone convergence theorem.

Since $T_{1} \neq \emptyset, m<\infty$, thus $t_{0} \geq m$, and $p_{m}=p_{m_{+}}$. In case $m>-\infty$, it must be that $\phi^{\prime}\left(k_{m}^{0}\right)=\infty$, which is only possible if $f^{\prime}(0)=\infty$, hence implying $k_{m}^{0}=0$. Then, for $t<m, p_{t}\left(c_{t}^{1}-c_{t}^{0}\right)$ is either 0 or $\pm \infty$. So for the integrals to be well-defined and $>0$, one must have $c_{t}^{1} \geq c_{t}^{0}$ for $-\infty<t<m$. If strict inequality holds on a set of positive measure there, then letting $c_{t}^{2}=c_{t}^{1}$ for $t<m$ and $=c_{t}^{0}$ else yields a feasible path, since $k_{m}^{0}=0 \leq k_{m}^{1}$. So $c^{2}$ shows that $c^{0}$ is not efficient: henceforth we can assume that $p_{t}=\infty \Rightarrow c_{t}^{1}=c_{t}^{0}$. Also, $k_{m}^{1}=k_{m}^{0}=0$ since otherwise, if $k_{m}^{1}>0$, it would have been possible to increase $c^{1}$ on an open interval just before $m$ (by continuity of $k^{1}$ ), hence contradicting the efficiency of $c^{0}$.

Clearly, $M>-\infty$. Similarly, if $M<\infty$ then $k_{M}^{0}=0$, and one can assume $k_{M}^{1}=0$. Indeed, first, $p_{M_{+}}=0$ implies $k_{M}^{0}=0$ and $\phi^{\prime}(0)=\infty$. If, to the contrary, $k_{M}^{1}>0$, then, by continuity of $k^{i}$, for some $\left.T \in\right] m, M\left[\right.$ it should be that $k_{t}^{1}>k_{t}^{0}$ for all $t \in\left[T, M\right.$ [, which implies it is feasible to increase consumption over $c^{1}$ in $\left[T, M\left[\right.\right.$; extending this then with $c^{0}, k^{0}$ after $M$ yields another $c^{1}, k^{1}$ for which our claim $k_{M}^{1}=0$ holds.

Let $\bar{a}=\max \{a, m\}, \bar{b}=\min \{b, M\}$. Then, $\liminf _{b,-a \rightarrow \infty} I_{a, b}=\liminf _{b,-a \rightarrow \infty} I_{\bar{a}, \bar{b}}$. Thus $m<M$ by positivity of the liminf. If $a \leq m$ and $M \leq b$, since the integral $I_{a, b}$ is well-defined and $>0$, the integrand is minorised by some Lebesgue integrable function on $[m, M]$; hence by the monotone convergence theorem $I_{m+\varepsilon_{a}, M-\varepsilon_{b}} \rightarrow I_{m, M}$, and thus $\lim \inf _{\varepsilon_{a}, \varepsilon_{b} \searrow 0} I_{m+\varepsilon_{a}, M-\varepsilon_{b}}=I_{m, M}>0$. So, in any case, $\lim \inf _{a \searrow m, b \succ M} I_{a, b}>0$. 
Step 2. There is $T \in] m, M[$, and $\varepsilon:] m, M[\rightarrow \mathbb{R}$ such that either

$$
\begin{array}{llll}
\text { case } A: & 0<\varepsilon_{t}<k_{t}^{0}-k_{t}^{1} \leq \kappa & \text { for } & T \leq t<M \text { or } \\
\text { case } B: & 0<-\varepsilon_{t}<k_{t}^{1}-k_{t}^{0} \leq \kappa & \text { for } & m<t \leq T
\end{array}
$$

with $k^{0}, k^{1}$ and $\varepsilon$ locally absolutely continuous on $] m, M[$.

Proof. By remark $2, k_{t}^{\prime} \leq \phi\left(k_{t}\right)+\Omega_{t}-c_{t}$. For $\left(k^{0}, c^{0}\right)$, there is no loss to assume that it holds as equality i.e., that no free-disposal is occurring, else $c^{0}$ can just be increased there, thus finishing the proof. Also we can assume there is no free-disposal of capital, i.e., a negative singular part $k^{s}$ of $k$, else, with $k^{a}=k-k^{s}$ one still has $k_{t}^{a \prime}=\phi\left(k_{t}\right)+\Omega_{t}-c_{t}^{0}$ a.e., and now $k^{a}>k$ on an open set, so the excess capital can just be disinvested and consumed there. Thus one can assume $k^{0}$ is locally absolutely continuous and $k_{t}^{0 \prime}=\phi\left(k_{t}^{0}\right)+\Omega_{t}-c_{t}^{0}$. Similarly for $k^{1}$ and $c^{1}$.

Use those equations to replace $c^{0}$ and $c^{1}$ in $I_{a, b}$, then

$$
\liminf _{a \searrow m, b \nearrow M} \int_{a}^{b} p_{t}\left[\phi\left(k_{t}^{1}\right)-\phi\left(k_{t}^{0}\right)-k_{t}^{1 \prime}+k_{t}^{0 \prime}\right] d t>0
$$

$\int_{a}^{b} p_{t}\left[k_{t}^{0 \prime}-k_{t}^{1 \prime}\right] d t$ can be integrated by parts for $a>m$ and $b<M$, since $p$ and the $k^{i}$ are absolutely continuous on $[a, b]$, yielding $p_{b}\left(k_{b}^{0}-k_{b}^{1}\right)-p_{a}\left(k_{a}^{0}-k_{a}^{1}\right)-\int_{a}^{b}\left(k_{t}^{0}-k_{t}^{1}\right) d p_{t}$. By eq. $2, d p_{t}=-p_{t} \phi^{\prime}\left(k_{t}^{0}\right) d t$.

$\liminf _{a \searrow m, b \nearrow M} \int_{a}^{b} p_{t}\left[\phi\left(k_{t}^{1}\right)-\phi\left(k_{t}^{0}\right)-\left(k_{t}^{1}-k_{t}^{0}\right) \phi^{\prime}\left(k_{t}^{0}\right)\right] d t-p_{b}\left(k_{b}^{1}-k_{b}^{0}\right)+p_{a}\left(k_{a}^{1}-k_{a}^{0}\right)>0$

Let then $\eta_{t}=-p_{t}\left[\phi\left(k_{t}^{1}\right)-\phi\left(k_{t}^{0}\right)-\left(k_{t}^{1}-k_{t}^{0}\right) \phi^{\prime}\left(k_{t}^{0}\right)\right], \geq 0$ by concavity of $\phi$, and, with $m<\tau<M, H_{t}=p_{t}\left(k_{t}^{1}-k_{t}^{0}\right)+\int_{\tau}^{t} \eta_{s} d s$ : $\limsup _{a \searrow m, b} \nearrow_{M}\left(H_{b}-H_{a}\right)<0$. Thus either $\lim \sup _{t}{ }_{M} H_{t}<0$ or $\left.\liminf _{t \searrow m} H_{t}>0: \exists T \in\right] m, M[$ s.t. either $\forall t: T \leq t<M, H_{t}<0$ or $\forall t: m<t \leq T, H_{t}>0$.

By step $1,0<p_{t}<\infty$ for $\left.t \in\right] m, M\left[\right.$. Thus we can divide by $p_{t}$ : let $\varepsilon_{t}=\left(p_{t}\right)^{-1} \int_{\tau}^{t} \eta_{s} d s$; then for some $\left.T \in\right] m, M\left[\right.$, either $k_{t}^{1}-k_{t}^{0}+\varepsilon_{t}<0 \forall t \in[T, M[$, or $k_{t}^{1}-k_{t}^{0}+\varepsilon_{t}>0$ for $\left.\left.t \in\right] m, T\right]$. Take also $T>\tau$ in the former case and $T<\tau$ else, so $\varepsilon_{t}$ is resp. $\geq 0$ and $\leq 0$. Also, the inequalities imply that in each case $\left|\varepsilon_{t}\right|<\left|k_{t}^{i}\right|$, which is $\leq \kappa$ by [16, prop. 1.a], so $\eta_{t}$ is locally integrable, and the integral is locally absolutely continuous in $t$. Further, since $p_{t}$ is locally absolutely continuous on $] m, M\left[\right.$, so is $\varepsilon_{t}$. Next, in the first case $\varepsilon>0$ in $\left[T, M\left[\right.\right.$, since else $\varepsilon_{t_{0}}=0$ for some $t_{0} \in\left[T, M\left[\right.\right.$ and $k_{t_{0}}^{1}-k_{t_{0}}^{0}+\varepsilon_{t_{0}}<0$ imply $k^{1} \neq k^{0}$ in a neighbourhood of $t_{0}$ (by continuity of $k^{0}, k^{1}$ ) which implies $\eta>0$ there by strict concavity of $f$, hence contradicting $\varepsilon_{t_{0}}=0$. Similarly, $\left.\left.\varepsilon_{t}<0 \forall t \in\right] m, T\right]$.

Step 3. The differential equation

$$
k_{t}^{\prime}=\phi\left(k_{t}\right)+\Omega_{t}-c_{t}^{0}-C, \quad C>0
$$

with initial condition $k_{T}=k_{T}^{0}$ has a unique (Caratheodory) solution ${ }^{7}$ on an interval ]$t_{1}, t_{2}[$ such that $T \in] t_{1}, t_{2}\left[\right.$ and either $t_{i}=\infty$ or $\lim _{t \rightarrow t_{i}} k_{t}=0$ for $i=1,2$.

Proof. Let $h_{t}=\int_{0}^{t}\left(\Omega_{s}-c_{s}^{0}-C\right) d s$. If $f^{\prime}(0)\left(=\phi^{\prime}(0)\right)=\infty$ then any feasible capital path is strictly positive a.e. inside interval $T_{1}$ (since $\phi^{\prime}\left(k_{s}\right)$ has to be integrable there), so choosing a slightly smaller $T$ if needed assures $k_{T}>0$. Then let $\mathscr{D}=\left\{(x, t) \in \mathbb{R}^{2} \mid x+h_{t}>0\right\}$, otherwise, (if $f^{\prime}(0)<\infty$ ), let $\mathscr{D}=\mathbb{R}^{2}$ and extend $f$ to, say, $f(x)=f(0)+x f^{\prime}(0)$ for $x \leq 0$ (thus, to be continuous and Lipschitz of $\mathscr{D})$. Further, in both cases on the corresponding domain $\mathscr{D}$, define $G(x, t)=\phi\left(x+h_{t}\right)$. The differential equation $x_{t}^{\prime}=G\left(x_{t}, t\right)$ with initial condition

\footnotetext{
${ }^{7}$ I.e., a locally absolutely continuous function $k_{t}$ satisfying the initial condition and s.t. plugging $k_{t}$ and $k_{t}^{\prime}$ into the differential equation yields equality a.e. It is easy to prove that there exists no classical solution, except if $\Omega_{t}-c_{t}^{0}$ is everywhere the derivative of its (own) primitive.
} 
$x_{T}=k_{T}^{0}-h_{T}$ has a unique (classical) solution on an interval $] t_{1}, t_{2}$ [ such that $T \in] t_{1}, t_{2}$ [ and either $t_{i}=\infty$ or $\lim _{t \rightarrow t_{i}} x_{t}+h_{t}=0$ for $i=1,2$. Indeed, on $\mathscr{D}, G(x, t)$ is continuous, and locally Lipschitz in the first argument, since for any $x, y \geq x_{0}>0,|G(x, t)-G(y, t)|=\left|\phi\left(x+h_{t}\right)-\phi\left(y+h_{t}\right)\right| \leq\left(f^{\prime}\left(x_{0}\right)+R\right)|x-y|$ by concavity of $f$. Further, any solution to that differential equation is locally bounded at any finite $t$ : indeed, by the differential equation and the triangular inequality $\left|x_{t}\right| \leq\left|x_{T}\right|+\int_{T}^{t}\left|G\left(x_{z}, z\right)\right| d z$, and since $\phi$ is continuous, it attains the supremum of its absolute value, $\hat{\phi}$, between $T$ and $t$, so the integral is majorised by $|T-t| \hat{\phi}$. Hence $x_{t}$ exists and is unique on $] t_{1}, t_{2}[$, by claim 1 in App. A.

Let $k_{t}=x_{t}+h_{t}$. Then $k_{T}=k_{T}^{0}, k$ is locally absolutely continuous, and satisfies the differential equation (3) wherever $h_{t}$ is differentiable with $h_{t}^{\prime}=\Omega_{t}-c_{t}^{0}-C$, thus, a.e. So $k$ is a Caratheodory solution of (3). Conversely, for any Caratheodory solution $k$ of $(3)$, let $x_{t}=k_{t}-h_{t}$. Then $x_{t}^{\prime}=G\left(x_{t}, t\right)$ a.e., and this right hand member is continuous, so, since $x$ is locally absolutely continuous, it coincides with the primitive of the right hand member: $x$ is $C^{1}$, and the equation $x_{t}^{\prime}=G\left(x_{t}, t\right)$ holds everywhere. Hence uniqueness of $x$, by the argument above - and thus uniqueness of $k$.

Step 4. $k_{t}>k_{t}^{0}$ on $\left[t_{1}, T\left[; k_{t}<k_{t}^{0}\right.\right.$ on $\left.] T, t_{2}\right] ; t_{1}=-\infty$.

Proof. Let $\xi_{t} \stackrel{\text { def }}{=} k_{t}-k_{t}^{0}$. Since $k_{t}^{0 \prime}=\phi\left(k_{t}^{0}\right)+\Omega_{t}-c_{t}^{0}$, and $k$ satisfies (3), and since $k$ and $k_{0}$ are locally absolutely continuous, $\xi_{t}=C(T-t)+\int_{T}^{t}\left[\phi\left(k_{s}\right)-\phi\left(k_{s}^{0}\right)\right] d s$, $\forall t \in\left[t_{1}, t_{2}\right]$ by continuity, when defining $k$ by continuity as 0 (cf. def. of $t_{i}$ ) at $t_{i}$ if $t_{i} \neq \infty$. Then for any $t \in\left[t_{1}, t_{2}\right]$, using a one-sided derivative if $t=t_{i} \neq \infty$, $\xi_{t}=0 \Rightarrow k_{t}=k_{t}^{0}$, so $\xi^{\prime}(t)$ exists and $=-C<0$. Thus $\xi$ can have at most one zero in $\left[t_{1}, t_{2}\right]$. But $\xi_{T}=0$, so $k_{t}<k_{t}^{0}$ on $\left.] T, t_{2}\right]$ and $k_{t}>k_{t}^{0}$ on $\left[t_{1}, T\left[\right.\right.$, so, $t_{1}=-\infty$.

Step 5. Let $T^{\prime}$ be $\inf \left\{t \geq T: k_{t}=k_{t}^{0}-\varepsilon_{t}\right\}$ in case $A$, and $\sup \left\{t \leq T: k_{t}=k_{t}^{0}-\varepsilon_{t}\right\}$ in case $B$. In case $A, T^{\prime} \leq t_{2} \leq M$ and $t_{2}<\infty$ implies both inequalities are strict. In case $B, m \leq T^{\prime}$ and if $m>-\infty$, the inequality is strict.

Proof. In case A, first, $t_{2} \leq M$ by step 4 and since both $k, k^{0}$ are continuous on $\left[T, t_{2}\right]$ and $\left[T, M\right.$ [ correspondingly. So if $t_{2}<\infty$ then $t_{2}<M$. Then, since $k_{t_{2}-}=0<k_{t}^{0}-\varepsilon_{t}$ for $t \in\left[T, M\left[\right.\right.$ (by step 2), $k_{T}=k_{T}^{0}>k_{T}^{0}-\varepsilon_{T}$ and $k_{t}$ is continuous on $\left[T, t_{2}\right.$ [, it had to cross $k_{t}^{0}-\varepsilon_{t}$ (continuous on $] m, M\left[\right.$ ) on $\left[T, t_{2}[\right.$, hence $T^{\prime}<t_{2}$. Clearly, otherwise $T^{\prime} \leq t_{2}=\infty$.

In case B, similarly, if $m>-\infty, k_{m}^{0}=k_{m}^{1}=0$ implies that $\lim _{t \searrow m} \varepsilon_{t}=0$, so $k_{m}>0=k_{m}^{0}-\varepsilon_{m}$, but $k_{T}=k_{T}^{0}<k_{T}^{0}-\varepsilon_{T}$, so the statement follows by continuity of $k, k^{0}, \varepsilon$ on $\left.] m, T\right]$.

Step 6. The segment between $T$ and $T^{\prime}$ is of positive length.

Proof. By step 5, T, $T^{\prime}$ are between $t_{1}$ and $t_{2}$ in both cases. If $T^{\prime}=\infty$ in case A (or $=-\infty$ in case B), the claim holds because $-\infty<T<\infty$. Otherwise, it follows from continuity of $k$ on $\left[t_{1}, t_{2}\right]$ and $k_{T^{\prime}}=k_{T^{\prime}}^{0}-\varepsilon_{T^{\prime}} \neq k_{T}^{0}=k_{T}$, as $\varepsilon_{T^{\prime}} \neq 0$ by step 2 in each case.

Now we define the dominating path $k^{2}$ :

Step 7. Let $k^{2}=k^{0}$ beyond $[m, M] ; k^{2}=k$ (the Caratheodory solution of (3)) between $T$ and $T^{\prime}$ and, from $T^{\prime}$ till $M$ in case $A$, or $m$ in case $B, k_{t}^{2}=k_{t}^{0}-\varepsilon_{t}$. Then $k^{2}$ is well-defined, locally absolutely continuous (except possibly at $M$ and $m$ ) and non-negative.

Proof. For the segments beyond $[m, M]$ the statement is obvious. Since $T, T^{\prime}$ are in $\left[t_{1}, t_{2}\right]$ by step $5, k^{2}$ is well-defined strictly between $T$ and $T^{\prime}$, it is absolutely continuous there by step 3 . The same step also assures absolute continuity at 
$T \in] t_{1}, t_{2}\left[\right.$. Also, by step $5, t_{2}<\infty \Rightarrow T^{\prime}<t_{2}$, so in this case absolute continuity of $k$ at $T^{\prime}$, too follows from step 3. So, whenever $\left|T^{\prime}\right|<\infty, k^{2}$ is well defined and absolutely continuous between $T$ and $T^{\prime}$ including the endpoints.

If $m \neq-\infty, k_{m}^{0}=k_{m}^{1}=0$ implies that $\lim _{t \searrow m} \varepsilon_{t}=0$, so, if $T^{\prime}=m$, since $k_{t}^{0}<k_{t}^{2}<k_{t}^{0}-\varepsilon_{t}$ on $] m, T\left[\lim _{t \searrow m} k_{t}^{2}=0\right.$, so $k_{m}^{2}=0$ and $k^{2}$ can indeed be continued continuously with $k^{0}$ beyond $m$. Similarly, $k^{2}$ is continuous at $M<\infty$.

Finally, for the third segment ([T,$M$ [in case A and $\left.] m, T^{\prime}\right]$ in case B), the local absolute continuity of $k^{2}$ follows from that of $k^{0}$ and of $\varepsilon$, and $k_{t}^{2}=k_{t}^{0}-\varepsilon_{t}>0$ in each case. Further, if $M<\infty$ or if $m \neq-\infty$, the inequalities imply as before that $\varepsilon_{t}$ and $k_{t}^{2}$ converge to 0 when $t \nearrow M$ (resp. $t \searrow m$ ) : again $k_{m}^{2}=k_{M}^{2}=0$.

Step 8. There is a feasible capital-consumption path $\left(k^{2}, c^{2}\right)$ (with $k^{2}$ defined in step 7) that dominates $\left(k^{0}, c^{0}\right)$.

Proof. Let now $c^{2}$ satisfy equation $k_{t}^{2 \prime}=\phi\left(k_{t}^{2}\right)+\Omega_{t}-c_{t}^{2}$. Remains to show, for feasibility of $\left(k^{2}, c^{2}\right)$, that the initial condition (assumption 1) is satisfied, that $k^{2}$ is locally absolutely continuous at $m$ and $M$ (if either is finite) and that $c^{2} \geq 0$. Finally, to show that $c^{1}$ is inefficient we need that $c_{t}^{2} \geq c_{t}^{0}$ a.e. This last point will imply $c^{2} \geq 0$ and the local absolute continuity. Indeed, if $m \neq-\infty(M<\infty$ resp.), since $k_{t}^{2 \prime}=\phi\left(k_{t}^{2}\right)+\Omega_{t}-c_{t}^{2}$ and $\phi$ is bounded and $\Omega \in L_{1}^{\text {loc }}$, that the positive increments of $k^{2}$ are summable on $\left[m, T^{\prime}\right]$ ([T',M] resp.), hence $k^{2}$ being continuous is of bounded variation there: local absolute continuity of $k^{2}$ holds at $m(M)$ too.

The initial condition is satisfied, in the first case because initially $k_{t}^{2}=k_{t}^{0}$, and in the other because, if $m=-\infty, \forall t \leq T, k_{t}^{2} \leq k_{t}^{0}-\varepsilon_{t}<k_{t}^{1}$ and else $k_{t}^{2}=k_{t}^{0}$ for $t \leq m$.

Remains thus to show that $c_{t}^{2} \geq c_{t}^{0}$ a.e. This is obvious in the first 2 segments: recall on the second segment (of positive length by step 6) the inequality is strict. On the last one, $\left\{t: \phi^{\prime}\left(k_{t}^{0}\right)=\infty\right\}$ is negligible, we get, with all equalities taken in the a.e. sense, as derivatives of locally absolutely continuous functions:

$$
\begin{aligned}
c_{t}^{2}-c_{t}^{0} & =\phi\left(k_{t}^{2}\right)-\phi\left(k_{t}^{0}\right)-k_{t}^{2 \prime}+k_{t}^{0 \prime} \\
& =\phi\left(k_{t}^{2}\right)-\phi\left(k_{t}^{0}\right)+\varepsilon_{t}^{\prime} \\
& =\phi\left(k_{t}^{2}\right)-\phi\left(k_{t}^{0}\right)-\frac{p_{t}^{\prime}}{p_{t}} \varepsilon_{t}-\phi\left(k_{t}^{1}\right)+\phi\left(k_{t}^{0}\right)+\left(k_{t}^{1}-k_{t}^{0}\right) \phi^{\prime}\left(k_{t}^{0}\right) \\
& =\phi\left(k_{t}^{2}\right)-\phi\left(k_{t}^{1}\right)-\left(-\phi^{\prime}\left(k_{t}^{0}\right)\right)\left(k_{t}^{0}-k_{t}^{2}\right)+\left(k_{t}^{1}-k_{t}^{0}\right) \phi^{\prime}\left(k_{t}^{0}\right) \\
& =\phi\left(k_{t}^{2}\right)+\left(k_{t}^{1}-k_{t}^{2}\right) \phi^{\prime}\left(k_{t}^{0}\right)-\phi\left(k_{t}^{1}\right)
\end{aligned}
$$

To prove that the last expression is $\geq 0$ : our previous inequalities imply, if $t \geq T$, $k^{1}<k^{2}<k^{0}$, and if $m<t \leq T, k^{0}<k^{2}<k^{1}$. So in any case, to minimise the expression over $k^{0}$ means setting $k^{0}=k^{2}$; hence the result by concavity of $\phi$.

\section{A SUFFICIENT CONDITION FOR ZERO NET ASSETS}

Since long ([13], [19]) the literature has alluded to a possible connection between (Pareto) efficiency and the amount of net assets the difference between aggregate consumer savings and the value of capital, in terms of Arrow-Debreu prices. ${ }^{8}$ As is shown in [16, thm.2], net assets are constant in any equilibirum (of the model adopted here). In GRE the constant is typically different from zero, whereas in a pure BGE (inefficient for a.e. capital share parameter $\alpha$ in the Cobb-Douglas production case) it is zero by [16, cor. 16].

Here we provide a sufficient condition for net assets to be zero in any equilibrium.

\footnotetext{
${ }^{8}$ Cf. [16, def. 4]. Cass and Yaari's "real money balances" are equivalent to net assets in our model. In both models if real money balances (net assets) are positive at some point in equilibrium, they remain so asymptotically, as $t \rightarrow \infty$.
} 
Lemma 1. In an equilibrium where $p^{C}=p^{I}=p^{Y}(=p), K_{t}>0$ and $\inf _{t} p_{t} L_{t}=0$, net assets are zero, $m=0$.

Proof. By [16, def. 4], $m_{t}$ is the difference between net consumer savings, $S_{t}$ and aggregate value of capital, $p_{t} K_{t}\left(=p_{t} L_{t} k_{t}\right)$. Net savings can be represented, by Fubini, ([16, lemma 14]) and notation 2.1, as $S_{t}=\int_{0}^{1} \int_{t+s-1}^{t} L_{z} p_{z}\left[\Xi_{z, s}-c_{z, s}^{\odot}\right] d z d s$, where $\Xi(t, s) \stackrel{\text { def }}{=} E_{t, s}+\left(f\left(k_{t}\right)-f^{\prime}\left(k_{t}\right) k_{t}\right) \varphi_{s}$.

Next, by [16, lemma 10.a5], $p_{t} L_{t}=L_{0} p_{0} e^{\int_{0}^{t} \mathfrak{r}_{v} d v}$, and by [16, thm. 2], net assets are constant in any such equilibrium, so

$$
m=L_{0} p_{0} e^{\int_{0}^{t-1} \mathfrak{r}_{v} d v}\left(\int_{0}^{1} \int_{t-1+s}^{t} e^{\int_{t-1}^{z} \mathfrak{r}_{v} d v}\left[\Xi_{z, s}-c_{z, s}^{\odot}\right] d z d s-e^{\int_{t-1}^{t} \mathfrak{r}_{u} d u} k_{t}\right) ;
$$

Since $\mathfrak{r}_{t} \leq R, \int_{t-1}^{t} \mathfrak{r}_{u} d u \leq R$ and since $t-z \leq 1, \int_{t-1}^{z} \mathfrak{r}_{u} d u \leq|z-t+1| R \leq R$, hence the term in the parenthesis is majorised by $e^{R}\left(\int_{0}^{1} \int_{t-1+s}^{t}\left[\Xi_{z, s}-c_{z, s}^{\odot}\right] d z d s-k_{t}\right)$. Take its absolute value, apply the triangular inequality for the term in the brackets, majorise $\left|\int\right|$ by $\int|\cdot|$, extend the integral to $0 \leq s \leq 1 ; t-1 \leq z \leq t$, use Fubini to change the order of integration, and the triangular inequality for the integrand. Then (1) implies

$$
\left|\frac{m}{p_{0} L_{0} e^{R}}\right| \leq e^{\int_{0}^{t-1} \mathfrak{r}_{v} d v}\left(\int_{t-1}^{t} \int_{0}^{1}\left[\left|\Xi_{z, s}\right|+\left|c_{z, s}^{\odot}\right|\right] d s d z+\left|k_{t}\right|\right)
$$

The inf of the first term, $e^{\int_{0}^{t-1} \mathfrak{r}_{v} d v}$, is zero by assumption. It is then left to show that the term in parenthesis in eq. 2 is bounded. Indeed,

$$
\int_{0}^{1}\left[\left|\Xi_{z, s}\right|+\left|c_{z, s}^{\odot}\right|\right] d s \leq\left|\Omega_{z}\right|+\left|f\left(k_{z}\right)-k_{z} f^{\prime}\left(k_{z}\right)\right|+\left|c_{z}\right|
$$

By assumption 2, $\|E\|_{\infty, 1}<\infty$ and so $\int_{t-1}^{t}\left|\Omega_{z}\right| d z$ is bounded, and by [16, prop. 1.c], the integral over the unit interval of $\left|c_{z}\right|$ is bounded as well. By [16, prop. 1.a], $k_{t} \geq 0$ is uniformly bounded, and so is $f\left(k_{t}\right) \geq 0$, further, since the wage rate is positive by [16, lemma 5.c], so is $f\left(k_{t}\right)-k_{t} f^{\prime}\left(k_{t}\right)$ by [16, lemma 10.a3] and since $k_{t} f^{\prime}\left(k_{t}\right) \geq 0$, $f\left(k_{t}\right)-k_{t} f^{\prime}\left(k_{t}\right)$ is bounded too, and so the conclusion follows.

\section{Concusions}

(Classical) Pareto efficiency of the GRE (our first theorem) for the OG economy with production has not been established before, to the best of our knowledge.

The second theorem, a necessary and sufficient condition for (strong) efficiency, is a generalisation of the first theorem in [10].

In his path-breaking work (analysing an OG model with production) Malinvaud [15] provides a sufficient criterion for (weak) efficiency: it is strikingly elegant, yet too permissive, requiring just the existence of price under which firms minimize costs and the value of (fully depreciating) capital to converge to zero as the time extends to the infinite future. Notice this is implied by the hypothesis of our lemma 1 for a (reduced) economy with a fixed population and without productivity growth $\left(L_{t}=\right.$ $\left.L_{0}\right)$. Cass [7] offered a necessary and sufficient condition for efficiency in a smooth neoclassical model, which requires the infinite sum (from time zero on) of $f^{\prime}\left(k_{t}\right)$ along a feasible path to diverge (thus, excluding paths with "over-accumulation of capital"). Balasko and Shell [2] provide a counter-part of the criterion for a smooth OG model with pure exchange, it is commonly referred to as "Cass criterion" ([4], $[6])$ : the infinite sum from some point on of the reciprocals of prices should diverge 
for a path to be efficient. All the above criteria mark as efficient all BGE with $\mathfrak{r}=R-f^{\prime}(k)<0$ which are clearly dominated by the GRE. ${ }^{9}$

\section{Appendix A. The maximal solution of a Differential Equation.}

Claim 1. For $\mathscr{D}$ open in $\mathbb{R}^{2}$, let $G: \mathscr{D} \rightarrow \mathbb{R}$ be continuous, and Lipschitz in the first argument. For $\left(x_{0}, t_{0}\right) \in \mathscr{D}$, the differential equation $x_{t}^{\prime}=G\left(x_{t}, t\right)$ has a unique solution for $t \in] t_{-}, t_{+}\left[,\left(t_{-}<t_{0}<t_{+}\right)\right.$, where $t_{-}>-\infty \Rightarrow \lim _{t \searrow_{-} t_{-}} x_{t}$ is the point at infinity in the one-point compactification of $\mathscr{D}$, and similarly at $t_{+}$.

Proof. By [11, thm. 10.4.5 pp. 289], there exists a unique solution over some interval ]$t_{-}, t_{+}[$. Take then a solution with a maximal such interval. If the claim would not hold, there would be a sequence $t_{n} \searrow t_{-}$such that $x_{t_{n}} \rightarrow \bar{x},\left(\bar{x}, t_{-}\right) \in \mathscr{D}$. Then $\exists \varepsilon>0: G$ is bounded on $[\bar{x}-\varepsilon, \bar{x}+\varepsilon] \times\left[t_{-}-\varepsilon, t_{-}+\varepsilon\right] \subseteq \mathscr{D}$, say $|G(x, t)| \leq M$. Then on $\left[t_{-}, t_{-}+\varepsilon / M\right]$ one must have $x_{t}$ in this rectangle, thus $\left|G\left(x_{t}, t\right)\right| \leq M$, so $x_{t}$ has bounded variation, and thus has a right-hand limit at $t_{-}$, which can only be $\bar{x}$ : $\lim _{t \searrow t_{-}} x_{t}=\bar{x}$. Let thus $x_{t_{-}} \stackrel{\text { def }}{=} \bar{x}$; by continuity of $G$ conclude first that $x_{t}^{\prime} \rightarrow G\left(x_{t_{-}}, t_{-}\right)$, next that $G\left(x_{t_{-}}, t_{-}\right)$is indeed the right-hand derivative of $x_{t}$ at $t_{-}$: we have a solution on $\left[t_{-}, t_{+}\left[\right.\right.$. Taking now $\left(x_{t_{-}}, t_{-}\right) \in \mathscr{D}$ as new initial point yields then by [11, thm. 10.4 .5 pp. 289] a solution on some larger interval, contradicting the maximality assumption.

\section{REFERENCES}

[1] Allais, M. (1947): Economie et intérêt. Imprimerie Nationale, Paris.

[2] Balasko, Y. and K. Shell (1980): "The overlapping generations model, I: The case of pure exchange without money". Journal of Economic Theory, vol. 23(3), pp. 281-306.

[3] Benveniste, L. and D. CAss (1986): "On the existence of optimal stationary equilibria with a fixed supply of fiat money: I. The case of a single consumer". Journal of Political Economy, vol. 94, pp. 402-417.

[4] Benveniste, L.M. (1986): "Pricing optimal distributions to overlapping generations: A corollary to efficiency pricing". The Review of Economic Studies, vol. 53(2), pp. 301-306.

[5] Burke, Jonathan L. (1995): "Existence of a Pareto-optimal equilibrium in nearly-stationary overlapping-generations economies". Economic Theory, vol. 5, pp. 247-261.

[6] — (1999): "The robustness of optimal equilibrium among overlapping generations". Economic Theory, vol. 14, pp. 311-329.

[7] CAss, D. (1972): "On capital overaccumulation in the aggregative, neoclassical model of economic growth: A complete characterization". Journal of Economic Theory, vol. 4(2), pp. 200-223, doi:10.1016/0022-0531(72)90149-4. URL http: //www.sciencedirect.com/science/article/pii/0022053172901494.

[8] — (1992): "On the existence of an optimal stationary equilibrium with a finite supply of fiat money. II: The case of many consumers with arbitrary lifetimes". Manuscript.

[9] Cass, D., M. OKuno, and I. Zilcha (1979): "The role of money in supporting the Pareto optimality of competitive equilibrium in consumption-loan type models". Journal of Economic Theory, vol. 20, pp. 41-80.

[10] CASs, D. and M. E. YAARI (1967): "Individual Saving, Aggregate Capital Accumulation, and Efficient Growth". In Essays on the Theory of Optimal Economic Growth, edited by ShelL, K., pp. 233-268, MIT Press, Cambridge (MA).

\footnotetext{
${ }^{9}$ Existence of a transfer $\left(E_{s}\right)$ to support such BGE in this model is guaranteed by [16, cor. 14].
} 
[11] Dieudonné, J.A., D. Huet, and M.G. Julia (1972): Éléments d'analyse: Fondements de l'analyse moderne, vol. 1. Gauthier-Villars, Paris.

[12] Duc, Francois and Christian Ghiglino (1998): "Optimality of barter steady states". Journal of Economic Dynamics and Control, vol. 22, pp. 1053-1067.

[13] Gale, David (1973): "Pure Exchange Equilibrium of Dynamic Economic Models". Journal of Economic Theory, vol. 6, pp. 12-36.

[14] Ghiglino, C. and M. Tvede (1995): "No-trade and uniqueness of steady states". Journal of Economic Dynamics and Control, vol. 19(3), pp. 655-661.

[15] Malinvaud, E. (1953): "Capital accumulation and efficient allocation of resources". Econometrica, pp. 233-268.

[16] Mertens, Jean-François and Anna Rubinchik (2012): "Equilibria in an overlapping generations model with transfer policies and exogenous growth". Working Paper 2012/5, University of Haifa, Israel.

[17] - (2012): "Intergenerational equity and the discount rate for policy analysis". Macroeconomic Dynamics, vol. 16, pp. 61-93. URL http://journals. cambridge.org/repo_A84Ar9FF.

[18] OKuno, M. and I. Zilcha (1983): "Optimal steady-state in stationary consumption-loan type models". Journal of Economic Theory, vol. 31, pp. 355363.

[19] Samuelson, Paul A. (1958): "An Exact Consumption-Loan Model of Interest with or without the Social Contrivance of Money". The Journal of Political Economy, vol. 66(6), pp. 467-482. 


\section{Recent titles \\ CORE Discussion Papers}

2011/64. Florian MAYNERIS and Sandra PONCET. French firms at the conquest of Asian markets: the role of export spillovers.

2011/65. Jean J. GABSZEWICZ and Ornella TAROLA. Migration, wage differentials and fiscal competition.

2011/66. Robin BOADWAY and Pierre PESTIEAU. Indirect taxes for redistribution: Should necessity goods be favored?

2011/67. Hylke VANDENBUSSCHE, Francesco DI COMITE, Laura ROVEGNO and Christian VIEGELAHN. Moving up the quality ladder? EU-China trade dynamics in clothing.

2011/68. Mathieu LEFEBVRE, Pierre PESTIEAU and Grégory PONTHIERE. Measuring poverty without the mortality paradox.

2011/69. Per J. AGRELL and Adel HATAMI-MARBINI. Frontier-based performance analysis models for supply chain management; state of the art and research directions.

2011/70. Olivier DEVOLDER. Stochastic first order methods in smooth convex optimization.

2011/71. Jens L. HOUGAARD, Juan D. MORENO-TERNERO and Lars P. ØSTERDAL. A unifying framework for the problem of adjudicating conflicting claims.

2011/72. Per J. AGRELL and Peter BOGETOFT. Smart-grid investments, regulation and organization.

2012/1. Per J. AGRELL and Axel GAUTIER. Rethinking regulatory capture.

2012/2. Yu. NESTEROV. Subgradient methods for huge-scale optimization problems.

2012/3. Jeroen K. ROMBOUTS, Lars STENTOFT and Francesco VIOLANTE. The value of multivariate model sophistication: An application to pricing Dow Jones Industrial Average options.

2012/4. Aitor CALO-BLANCO. Responsibility, freedom, and forgiveness in health care.

2012/5. Pierre PESTIEAU and Grégory PONTHIERE. The public economics of increasing longevity.

2012/6. Thierry BRECHET and Guy MEUNIER. Are clean technology and environmental quality conflicting policy goals?

2012/7. Jens L. HOUGAARD, Juan D. MORENO-TERNERO and Lars P. ØSTERDAL. A new axiomatic approach to the evaluation of population health.

2012/8. Kirill BORISSOV, Thierry BRECHET and Stéphane LAMBRECHT. Environmental maintenance in a dynamic model with heterogenous agents.

2012/9. Ken-Ichi SHIMOMURA and Jacques-François THISSE. Competition among the big and the small.

2012/10. Pierre PESTIEAU and Grégory PONTHIERE. Optimal lifecycle fertility in a Barro-Becker economy.

2012/11. Catherine KRIER, Michel MOUCHART and Abderrahim OULHAJ. Neural modelling of ranking data with an application to stated preference data.

2012/12. Matthew O. JACKSON and Dunia LOPEZ-PINTADO. Diffusion and contagion in networks with heterogeneous agents and homophily.

2012/13. Claude D'ASPREMONT, Rodolphe DOS SANTOS FERREIRA and Jacques THEPOT. Hawks and doves in segmented markets: A formal approach to competitive aggressiveness.

2012/14. Claude D'ASPREMONT and Rodolphe DOS SANTOS FERREIRA. Household behavior and individual autonomy: An extended Lindahl mechanism.

2012/15. Dirk VAN DE GAER, Joost VANDENBOSSCHE and José Luis FIGUEROA. Children's health opportunities and project evaluation: Mexico's Oportunidades program.

2012/16. Giacomo VALLETTA. Health, fairness and taxation.

2012/17. Chiara CANTA and Pierre PESTIEAU. Long term care insurance and family norms.

2012/18. David DE LA CROIX and Fabio MARIANI. From polygyny to serial monogamy: a unified theory of marriage institutions.

2012/19. Carl GAIGNE, Stéphane RIOU and Jacques-François THISSE. Are compact cities environmentally friendly?

2012/20. Jean-François CARPANTIER and Besik SAMKHARADZE. The asymmetric commodity inventory effect on the optimal hedge ratio. 


\section{Recent titles}

\section{CORE Discussion Papers - continued}

2012/21. Concetta MENDOLICCHIO, Dimitri PAOLINI and Tito PIETRA. Asymmetric information and overeducation.

2012/22. Tom TRUYTS. Stochastic signaling: Information substitutes and complements.

2012/23. Pierre DEHEZ and Samuel FEREY. How to share joint liability: A cooperative game approach.

2012/24. Pilar GARCIA-GOMEZ, Erik SCHOKKAERT, Tom VAN OURTI and Teresa BAGO D'UVA. Inequity in the face of death.

2012/25. Christian HAEDO and Michel MOUCHART. A stochastic independence approach for different measures of concentration and specialization.

2012/26. Xavier RAMOS and Dirk VAN DE GAER. Empirical approaches to inequality of opportunity: principles, measures, and evidence.

2012/27. Jacques H. DRĖZE and Erik SCHOKKAERT. Arrow's theorem of the deductible : moral hazard and stop-loss in health insurance.

2012/28 Luc BAUWENS and Giuseppe STORTI. Computationally efficient inference procedures for vast dimensional realized covariance models.

2012/29. Pierre DEHEZ. Incomplete-markets economies: The seminar work of Diamond, Drèze and Radner.

2012/30 Helmuth CREMER, Pierre PESTIEAU and Grégory PONTHIÈRE. The economics of longterm care: a survey.

2012/31 Mathieu VAN VYVE, Laurence A. WOLSEY and Hande YAMAN. Relaxations for two-level multi-item lot-sizing problem.

2012/32 Jean-François MERTENS and Anna RUBINCHIK. Equilibria in an overlapping generations model with transfer policies and exogenous growth.

2012/33 Jean-François MERTENS and Anna RUBINCHIK. Pareto optimality of the golden rule equilibrium in an overlapping generations model with production and transfers.

\section{Books}

G. DURANTON, Ph. MARTIN, Th. MAYER and F. MAYNERIS (2010), The economics of clusters Lessons from the French experience. Oxford University Press.

J. HINDRIKS and I. VAN DE CLOOT (2011), Notre pension en heritage. Itinera Institute.

M. FLEURBAEY and F. MANIQUET (2011), A theory of fairness and social welfare. Cambridge University Press.

V. GINSBURGH and S. WEBER (2011), How many languages make sense? The economics of linguistic diversity. Princeton University Press.

I. THOMAS, D. VANNESTE and X. QUERRIAU (2011), Atlas de Belgique - Tome 4 Habitat. Academia Press.

W. GAERTNER and E. SCHOKKAERT (2012), Empirical social choice. Cambridge University Press.

L. BAUWENS, Ch. HAFNER and S. LAURENT (2012), Handbook of volatility models and their applications. Wiley.

J-C. PRAGER and J. THISSE (2012), Economic geography and the unequal development of regions. Routledge.

M. FLEURBAEY and F. MANIQUET (2012), Equality of opportunity: the economics of responsibility. World Scientific.

J. HINDRIKS (2012), Gestion publique. De Boeck.

\section{CORE Lecture Series}

R. AMIR (2002), Supermodularity and complementarity in economics.

R. WEISMANTEL (2006), Lectures on mixed nonlinear programming.

A. SHAPIRO (2010), Stochastic programming: modeling and theory. 